BMJ Open

Respiratory

Research

\title{
Serial characterisation of monocyte and neutrophil function after lung resection
}

Richard O Jones, ${ }^{1,2}$ Mairi Brittan, ${ }^{1}$ Niall H Anderson, ${ }^{3}$ Andrew Conway Morris, ${ }^{1,4}$ John T Murchison, ${ }^{5}$ William S Walker, ${ }^{2}$ A John Simpson ${ }^{1,6}$

\section{ABSTRACT}

To cite: Jones RO, Brittan M, Anderson $\mathrm{NH}$, et al. Serial characterisation of monocyte and neutrophil function after lung resection. BMJ Open Resp Res 2014;1:e000045. doi:10.1136/bmjresp-2014000045

\section{- Additional material is available. To view please visit the journal (http://dx.doi.org/ 10.1136/bmjresp-2014- 000045)}

WSW and AJS are joint senior authors.

Received 18 May 2014 Revised 15 July 2014 Accepted 19 July 2014

\section{(1) CrossMark}

For numbered affiliations see end of article.

Correspondence to Professor A John Simpson; j.simpson@ncl.ac.uk
Objectives: The primary aim of this prospective study was to perform a comprehensive serial characterisation of monocyte and neutrophil function, circulating monocyte subsets, and bronchoalveolar lavage (BAL) fluid after lung resection. A secondary aim was to perform a pilot, hypothesis-generating evaluation of whether innate immune parameters were associated with postoperative pneumonia.

Methods: Forty patients undergoing lung resection were studied in detail. Blood monocytes and neutrophils were isolated preoperatively and at 6, 24 and $48 \mathrm{~h}$ postoperatively. BAL was performed preoperatively and immediately postoperatively. Monocyte subsets, monocyte responsiveness to lipopolysaccharide (LPS) and neutrophil phagocytic capacity were quantified at all time points. Differential cell count, protein and cytokine concentrations were measured in BAL. Pneumonia evaluation at $72 \mathrm{~h}$ was assessed using predefined criteria.

Results: After surgery, circulating subsets of classical and intermediate monocytes increased significantly. LPS-induced release of proinflammatory cytokines from monocytes increased significantly and by $48 \mathrm{~h} \mathrm{a}$ more proinflammatory profile was found. Neutrophil phagocytosis demonstrated a small but significant fall. Factors associated with postoperative pneumonia were: increased release of specific proinflammatory and antiinflammatory cytokines from monocytes; preoperative neutrophilia; and preoperative BAL cell count.

Conclusions: We conclude that postoperative lung inflammation is associated with specific changes in the cellular innate immune response, a better understanding of which may improve patient selection and prediction of complications in the future.

\section{INTRODUCTION}

In recent years, there has been increasing recognition of a complex innate immune response to insults such as sepsis or trauma. It is generally accepted that such processes induce not only a systemic inflammatory response syndrome, but also a compensatory anti-inflammatory response (CARS). ${ }^{12}$ The magnitude and duration of both processes appear to dictate clinical outcomes,

\section{KEY MESSAGES}

Lung resection is followed by a systemic innate immune response characterised by an increase in classical and intermediate monocytes, heightened pro-inflammatory responsiveness of monocytes, and reduced neutrophil phagocytic capacity.

- Within 2 hours of lung resection, an inflammatory response evolves in the contralateral lung.

Pre-operative and pre-operative characteristics of the innate cellular innate immune response appear to be associated with post-operative lung inflammation, though these require validation in future studies.

suggesting that a closely coordinated interaction between these apparently divergent responses is beneficial. ${ }^{3}$

These concepts have largely arisen from meticulous serial analysis of plasma cytokines. Far less is known about the innate cellular immune response in this context. Monocytes are known to infiltrate the lung in a diverse range of inflammatory lung conditions. ${ }^{4-7}$ Improved methods for phenotypic characterisation have led to clear demonstrations of distinct subsets of circulating murine monocytes, and a similar concept has emerged in humans with the identification of classical, non-classical and intermediate monocyte subsets. ${ }^{8-11}$ Monocyte deactivation has been associated with adverse outcomes in patients with sepsis. ${ }^{12-15}$ However, remarkably little is known about serial functional responses of monocytes and neutrophils under pathological conditions.

The aim of this study was therefore to perform the first detailed 'within-patient' characterisation of circulating cellular innate responses after lung resection. The setting of lung resection was chosen as there is a relatively consistent and controlled level of surgical trauma, and because thoracic surgery may be complicated by postoperative infection or acute lung injury (ALI). ${ }^{16-18}$ Secondary aims were: (1) to make preliminary analyses of 
whether innate immune dysfunction predicted postoperative complications (in the form of alveolar consolidation on chest X-ray (CXR) at $72 \mathrm{~h}$ ) and (2) to take the unique opportunity to study serial cellular responses in bronchoalveolar lavage (BAL) fluid from patients having lung resection.

\section{MATERIALS AND METHODS}

\section{Recruitment of patients}

Patients referred for thoracic surgery with proven or clinically suspected lung cancer were enrolled prospectively. They were excluded if there was evidence of metastatic disease on routine preoperative staging. All patients were surgically staged with bronchoscopy and videomediastinoscopy prior to lung resection. Informed, written consent was obtained from all patients.

\section{Blood sampling and preparation}

Venepuncture was performed preoperatively and at 6, 24 and $48 \mathrm{~h}$ postoperatively. Isolation of granulocytes and mononuclear cells from whole blood was performed at each time point using dextran sedimentation and percoll gradient extraction. ${ }^{19}$ Neutrophil purity in extracted granulocyte layers was consistently $>95 \%$. Monocytes were positively selected from the mononuclear layer using magnetic beads labelled with an anti-CD14 antibody (Miltenyi Biotec, Germany). Using this technique, the monocyte purity was consistently $>90 \%$.

\section{Flow cytometric analysis of monocyte subsets}

Five-colour immunofluorescent labelling of the mononuclear layer of freshly prepared blood was performed (using antibodies specific to HLA-DR, CD14, CD16, CCR2 and $\left.\mathrm{CX}_{3} \mathrm{CR} 1\right)$. An unstained sample and five single stains were used to compensate the data with a mixture sample containing all five antibodies used to identify monocyte subsets.

Monocytes were identified by gating on a cell population with the characteristic forward scatter and side scatter. Within this gate, HLA-DR-positive cells were identified, and relative CD14 and CD16 expression on these was used to identify the three principal human monocyte subsets (CD14++CD16- 'classical' monocytes; CD14++CD16+ 'intermediate' monocytes; and CD14 +CD16++ 'non-classical' monocytes). Antibodies to CCR2 and $\mathrm{CX}_{3} \mathrm{CR} 1$ were used to confirm that each of the three monocyte subsets (identified by the relative CD14/CD16 expression) had the characteristic expression profile for these receptors.

\section{Stimulation of monocytes with lipopolysaccharide}

Isolated monocytes were stimulated with lipopolysaccharide (LPS) from Escherichia coli 026:B6 (Sigma, UK), final concentration $154 \mathrm{ng} / \mathrm{mL}$, for $18 \mathrm{~h}$ in duplicate in a 24-well Costar plate (Corning, Amsterdam). Supernatants were collected and frozen at $-80^{\circ} \mathrm{C}$ until later analysis. Data were adjusted for total protein using the Bradford method (Sigma, UK).

\section{Phagocytosis by neutrophils}

Phagocytosis by freshly prepared neutrophils was evaluated by flow cytometric analysis of neutrophils exposed to serum-opsonised zymosan (from Saccharomyces cerevisiae) preconjugated with Alexa Fluor 594 (Invitrogen, UK). Following culture in suspension for $30 \mathrm{~min}$, addition of trypan blue (to quench extracellular fluorescence) and multiple washes, flow cytometric analysis was performed (BD Special Order LSR Fortessa, Becton Dickinson). The data were subsequently analysed using FlowJo Software (V.7.6.3; TreeStar Inc) with the unstained sample being used as the basis of the gating for positive phagocytosis.

\section{Bronchoalveolar lavage}

BAL was performed twice in each patient at least 1 week apart, thereby allowing any BAL-induced inflammation to recede. The first BAL was performed prior to mediastinoscopy, that is, before any form of surgical trauma, thus serving as a preoperative sample. The second BAL was performed immediately after lung resection and served as an early postoperative comparator sample. BAL was performed in the lung contralateral to the tumour on both occasions, and a different lobe was lavaged at the second BAL.

BAL was performed under general anaesthesia using a standardised technique. ${ }^{20}$ Quantification of cell counts, total protein and preparation of cell-free supernatant for later analysis were performed as described previously. ${ }^{20}$

\section{Cytokine analysis}

Serum and BAL cytokines were analysed using a commercially available Cytometric Bead Array kit (BD Human Inflammatory Cytokines Kit, Becton Dickinson Biosciences).

\section{Definition of postoperative pneumonia}

Pneumonia was predefined as new alveolar infiltrates on a CXR at $72 \mathrm{~h}$, reported by an experienced pulmonary radiologist blinded to study results, plus two from three of: temperature $>38.0^{\circ} \mathrm{C}$ or $<35.5^{\circ} \mathrm{C}$; white cell count $>11.0 \times 10^{9} / \mathrm{L}$ or $<4.0 \times 10^{9} / \mathrm{L}$; and positive microbiology on sputum culture.

\section{Statistical analysis}

Data were analysed using the Minitab 16 Software (Minitab Inc). Normality of distribution was assessed using the Anderson-Darling test. Non-normally distributed data were $\log _{10}$ transformed prior to further analysis. Postoperative changes were compared using the paired $\mathrm{t}$ test and groups compared using the two-sample Student $t$ test. A repeated measures ANOVA/ANCOVA model was created to incorporate analysis of variance attributable to time points and the pneumonia group. To determine relative risk, Youden's test was applied to 
parameters that were significantly higher in patients with pneumonia to establish the optimal cut-off for predicting pneumonia. The relative risk for each parameter was then determined using Fisher's test and the likelihood ratio calculated. Statistical significance was considered to be at the $\mathrm{p}<0.05$ level. The statistical programme GraphPad Prism 5 (GraphPad Software Inc) was used for this purpose.

\section{RESULTS}

\section{Patient characteristics}

Fifty-three patients consented to take part, of whom 13 were excluded ( 7 had lymph node involvement at mediastinoscopy, 2 withdrew consent postoperatively, 1 was deemed medically unfit for resection after staging and 3 were excluded for practical reasons precluding full sample collection). Demographic and clinical characteristics of the 40 patients analysed are shown in table 1 . Sixteen patients $(40 \%)$ had a preoperative diagnosis of chronic obstructive pulmonary disease. Surgical resection was performed whenever possible via video-assisted thoracoscopic surgery (VATS) lobectomy $(\mathrm{n}=26)$; otherwise, posterolateral thoracotomy $(\mathrm{n}=14)$ was performed. Seventeen patients $(42.5 \%)$ proved to have adenocarcinoma and 14 $(35.0 \%)$ had squamous cell carcinoma. Details of final histological diagnoses and staging are described in the online supplementary section (table S1).

\section{Response of circulating blood neutrophils and monocytes to lung resection}

As expected, a significant postoperative leucocytosis was observed ( $\mathrm{p}<0.0005$ by ANOVA modelling). This largely

\section{Table 1 Patient characteristics}

\begin{tabular}{|c|c|}
\hline Characteristic & $\begin{array}{l}\text { All patients } \\
n=40\end{array}$ \\
\hline Male, n (\%) & $23(57.5)$ \\
\hline Mean age, years (SD) & $66.9(7.8)$ \\
\hline Mean BMI, $\mathrm{kg} / \mathrm{m}^{2}$ (SD) & $26.6(4.3)$ \\
\hline Current smoker, $\mathrm{n}(\%)^{*}$ & $13(32.5)$ \\
\hline $\mathrm{FEV}_{1}, \%$ predicted (SD) & $87.3(23.8)$ \\
\hline FEV 1 /FVC, \% predicted (SD) & $88.5(13.9)$ \\
\hline $\mathrm{T}_{\mathrm{CO}}, \%$ predicted $(\mathrm{SD})$ & $72.0(21.9)$ \\
\hline $\mathrm{K}_{\mathrm{CO}}$, \% predicted (SD) & $78.1(17.6)$ \\
\hline Mean thoracoscore (SD) & $2.5(1.7)$ \\
\hline Inpatient mortality, n (\%) & $0(0)$ \\
\hline Median blood loss, mL (IQR) & $120(55-300)$ \\
\hline $\begin{array}{l}\text { Mean change in blood } \mathrm{Hb}, \mathrm{g} / \mathrm{L} \text { (between } \\
\text { pre-op and day } 1 \text { post-op values) (SD) }\end{array}$ & $13.8(10.0)$ \\
\hline Mean duration of surgery, minutes (SD) & $203(44.4)$ \\
\hline \multicolumn{2}{|c|}{$\begin{array}{l}\text { Thoracoscore is a validated scoring system used to predict } \\
\text { outcomes after thoracic surgery. }{ }^{21} \\
{ }^{*} \text { Current or ex-smoker }<2 \text { months duration. } \\
\text { BMI, body mass index; } \mathrm{FEV}_{1} \text {, forced expiratory volume in } 1 \mathrm{~s} \text {; } \\
\text { FVC, forced vital capacity; } \mathrm{Hb} \text {, haemoglobin; } \mathrm{K}_{\mathrm{co}} \text {, diffusing } \\
\text { capacity for carbon monoxide, corrected for lung volume; } \\
\text { Post-op, postoperative; Pre-op, postoperative; } \mathrm{T}_{\mathrm{co}} \text {, diffusing } \\
\text { capacity for carbon monoxide. }\end{array}$} \\
\hline
\end{tabular}

reflected a significant rise in blood neutrophils, but there was an associated significant rise in monocytes and a fall in lymphocytes $(p<0.0005$ for each cell type by ANOVA modelling).

Table 2 describes the effect of LPS on blood-derived monocytes. It should be emphasised that these data are for all cytokines released from circulating CD14-positive monocytes (ie, no inferences can be drawn in relation to secretion by monocyte subsets or to intracellular expression of the cytokines described). It should also be emphasised that each time point describes the effects of LPS stimulation-basal levels of cytokine secretion from preoperative monocytes that were not stimulated with LPS are shown in the online supplementary section (table S2). ANOVA modelling revealed a significant postoperative increase in IL-8 and tumour necrosis factor (TNF) release from monocytes stimulated with LPS $e x$ vivo (table 2). By $48 \mathrm{~h}$ IL-8, TNF and IL-6 secretion were increased significantly, and IL-10 secretion decreased significantly, suggesting a tendency towards monocytes exhibiting a more proinflammatory state of responsiveness.

The response in serum cytokines over the same time course was subtly different from the response of isolated monocytes to LPS. In serum, IL-6, IL-8 and IL-10 showed very similar trends, all rising gradually to $24 \mathrm{~h}$, and returning towards (but not back to) baseline by $48 \mathrm{~h}$ (all $\mathrm{p}<0.0005$ by ANOVA modelling; details are found in the online supplementary section, table S3). No significant differences were observed in the postoperative serum levels of IL-1 $\beta$, IL-12 or TNF.

Surgery was associated with a significant absolute increase in circulating populations of both classical and intermediate monocytes postoperatively, with a corresponding non-significant trend towards a reduction in the number of non-classical monocytes (figure 1A, B). The increase in classical and intermediate monocytes appeared to be established by $24 \mathrm{~h}$.

ANOVA modelling revealed a small but statistically significant postoperative reduction in the phagocytic capacity of neutrophils in suspension (the percentage of neutrophils ingesting 2 or more zymosan particles (expressed as median with IQR in parenthesis) was 84 $(73-91)$ at baseline, $84(68-90)$ at $6 \mathrm{~h}, 79(64-86)$ at $24 \mathrm{~h}$, and $79(63-87)$ at $48 \mathrm{~h}$; ANOVA for overall trend, $\mathrm{p}=0.047$ ).

\section{Pulmonary response to lung resection}

Surgery was associated with a significant increase in total leucocyte count, total protein and levels of IL-1 $\beta$, IL-6, IL-8 and IL-10 in BAL fluid retrieved from the contralateral lung in the immediate postoperative period (table 3). No evidence of infection was found in any of the BAL fluid cultures at this time point.

\section{Associations with postoperative pneumonia}

Fourteen patients $(35 \%)$ developed postoperative pneumonia according to the predefined criteria. There was 
Table 2 Ex vivo monocyte responsiveness to stimulation with LPS

\begin{tabular}{|c|c|c|c|c|c|}
\hline Cytokine & $\begin{array}{l}0 \mathrm{~h} \\
\mathrm{pg} / \mathrm{mL} / \mu \mathrm{g} \\
\text { Median (IQR) }\end{array}$ & $\begin{array}{l}6 \mathrm{~h} \\
\mathrm{pg} / \mathrm{mL} / \mu \mathrm{g} \\
\text { Median (IQR) }\end{array}$ & $\begin{array}{l}24 \mathrm{~h} \\
\mathrm{pg} / \mathrm{mL} / \mu \mathrm{g} \\
\text { Median (IQR) }\end{array}$ & $\begin{array}{l}48 \mathrm{~h} \\
\mathrm{pg} / \mathrm{mL} / \mu \mathrm{g} \\
\text { Median (IQR) }\end{array}$ & $\begin{array}{l}\text { ANOVA } \\
\text { p Value }\end{array}$ \\
\hline IL-8 & 1334 (915-1923) & $2325(1194-3059)^{\star *}$ & 2177 (1072-2734) & $2409(1420-2787)^{\star \star}$ & 0.034 \\
\hline IL-6 & 282 (130-362) & $391(170-580)$ & 395 (146-601) & $454(192-703)^{\star}$ & 0.358 \\
\hline TNF & $39.2(25.8-49.6)$ & $40.7(25.4-69.5)$ & $43.6(23.8-67.8)$ & $48.1(34.8-82.4)^{\star}$ & 0.028 \\
\hline IL-10 & 2.14 (1.18-3.52) & $2.61(0.88-5.07)$ & $1.96(1.02-3.36)$ & $1.63(0.78-2.77)^{*}$ & 0.122 \\
\hline IL-1 $\beta$ & $28.1(15.5-52.6)$ & $20.1(11.6-35.1)$ & $23.4(11.1-73.5)$ & 18.7 (10.8-32.7) & 0.291 \\
\hline IL-12 & $0.17(0.14-0.26)$ & $0.18(0.12-0.24)$ & $0.19(0.13-0.26)$ & $0.16(0.13-0.26)$ & 0.451 \\
\hline
\end{tabular}

Blood monocytes were isolated at each of the four perioperative time points and stimulated with LPS for $18 \mathrm{~h}$, ie, isolated monocytes were stimulated once with LPS - no cells received repeated application of LPS. Cytokine concentrations in supernatants were quantified. Data were adjusted for total protein content. Data are presented as medians and IQR. Data were analysed for trend by ANOVA; individual time points were assessed relative to the preoperative value by paired Student $t$ test, ${ }^{*} p<0.05$, ${ }^{* *} p<0.005 . n=40$ for all time points except $48 h$, where $n=38$. ANOVA, analysis of variance; LPS, lipopolysaccharide; TNF, tumour necrosis factor.

no significant difference in the rate of pneumonia according to which form of surgery was performed $(35 \%$ in patients undergoing VATS, $36 \%$ in patients having thoracotomy). No patients developed ALI/acute respiratory distress syndrome as measured by published criteria. $^{22}$ The only baseline clinical factor associated with a significant difference between those who did or did not develop pneumonia was the transfer factor for carbon monoxide $(60.9 \%$ predicted in those who developed pneumonia vs $77.9 \%$ predicted in those who did not, $\mathrm{p}=0.010$ ). Further details of baseline clinical characteristics for these two subgroups are found in the online supplementary section (table S4).

We went on to test whether individual innate immune parameters were associated with the development of postoperative pneumonia (table 4 panel A). In general, serum cytokines were not associated with pneumonia, and where they were, the proportional difference was small. Similarly, neutrophil phagocytosis was not associated with pneumonia. In contrast, preoperative cell counts and elements of monocyte responsiveness appeared to be associated with pneumonia.

To test this further, parameters identified in table 4 panel A were used to establish which had a significant relative risk for pneumonia (table 4 panel B). In this setting, relatively higher neutrophils in blood and leucocytes in BAL appeared to be associated with pneumonia. Interestingly, relatively higher anti-inflammatory responses in LPS-stimulated monocytes (IL-12 preoperatively and IL-10 at $24 \mathrm{~h}$ ) were associated with pneumonia, with IL-6 being the only proinflammatory cytokine to show an association.

\section{DISCUSSION}

While several studies have assessed the serum cytokine response after thoracic surgery, ${ }^{23-25}$ our findings provide a first detailed, serial characterisation of monocyte and neutrophil function after lung resection. Elective surgery provided a particularly useful setting in which to study innate immune characteristics in the lung and blood, given the opportunity to obtain a preoperative baseline. Our data suggest that classical monocytes are mainly responsible for postoperative monocytosis. Intermediate monocytes were also significantly increased, but interestingly non-classical monocytes appeared to be numerically and proportionally decreased relative to baseline, at least as far out as $48 \mathrm{~h}$. Similar numerical changes have been described after stroke. ${ }^{26}$ The fate of intermediate monocytes in this setting remains to be determined-some evidence exists for this population having inherent plasticity, with the capacity to become classical or non-classical monocytes depending on their environment. ${ }^{27} 28$

We also report a dynamic state of monocyte responsiveness to LPS, with IL-8 secretion increased within $6 \mathrm{~h}$, and a general tendency for a more proinflammatory cytokine responsiveness to LPS at $48 \mathrm{~h}$. The pattern of increased LPS-induced IL-8 secretion from monocytes postoperatively is broadly similar to that described in a smaller study of mononuclear cells from (non-thoracic) surgical patients, though we did not observe the increase in IL-1 $\beta$ described in that study. ${ }^{29}$ Certainly, our data suggest that, on average, monocytes have a heightened proinflammatory response to LPS in the postoperative period.

In this regard, a number of points are worth making. First, our data suggest that the monocyte response generates proinflammatory cytokines from an early stage. This is broadly reminiscent of the pattern described in serum. Second, it is intriguing that the relative risk for pneumonia was most closely associated with monocyte release of the anti-inflammatory cytokine IL-10 (table 4 panel B). With regard to associations with postoperative pneumonia, ours was very much a pilot, hypothesisgenerating study, and as such the sample size was too small (and not designed) to draw conclusions on whether a relative anti-inflammatory postoperative monocyte response is predictive of subsequent pneumonia. Nevertheless, IL-10 is differentially expressed by 
A

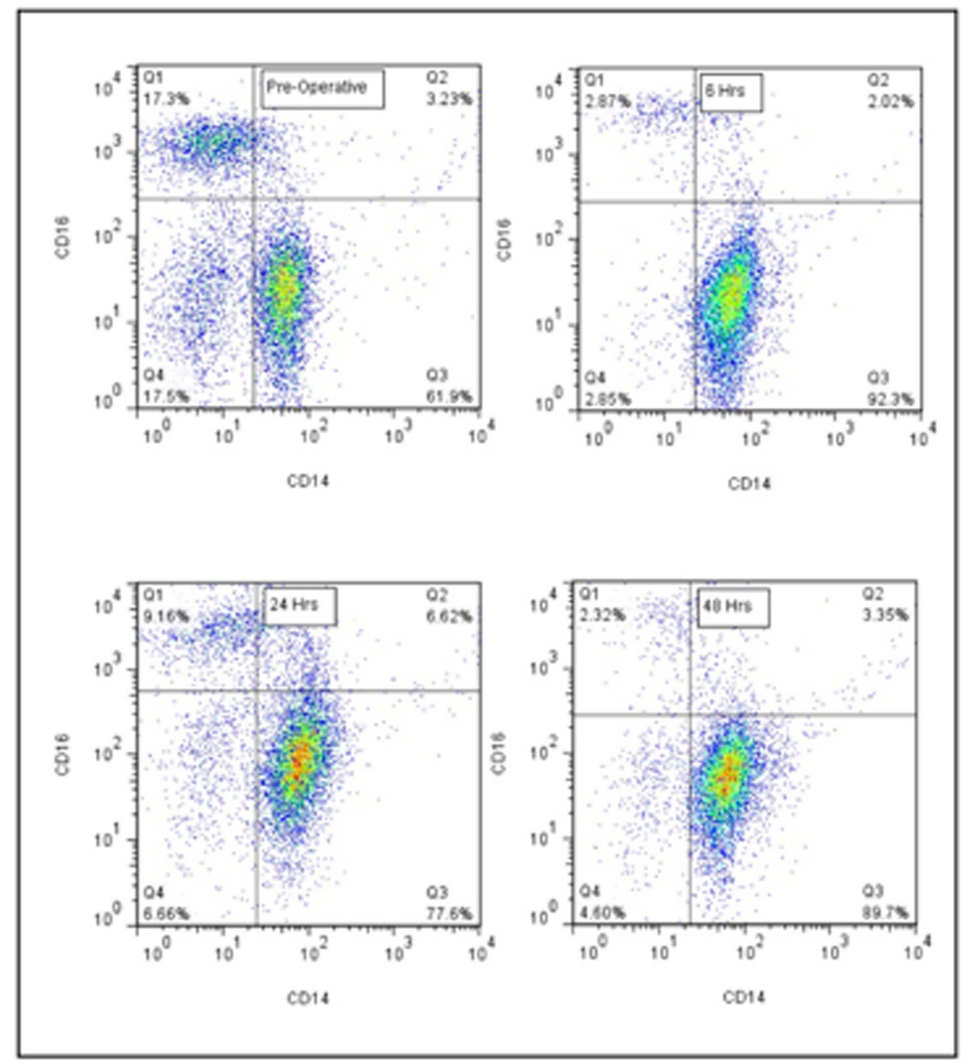

B

\begin{tabular}{|c|c|c|c|c|c|}
\hline Monocyte Subset & $\begin{array}{l}\text { Pre-Op } \\
\text { Median } \\
\text { Count } \\
\left(\times 10^{\circ} n\right)\end{array}$ & $\begin{array}{l}24 \mathrm{Hrs} \\
\text { Median } \\
\text { Count } \\
\left(\times 10^{\circ} / \mathrm{l}\right)\end{array}$ & $\begin{array}{l}48 \mathrm{Hrs} \\
\text { Median } \\
\text { Count } \\
\left(\times 10^{\circ} / 1\right)\end{array}$ & $\begin{array}{l}\text { p-value } \\
24 \mathrm{Hrs}\end{array}$ & $\begin{array}{l}\text { p-value } \\
48 \mathrm{Hrs}\end{array}$ \\
\hline $\mathrm{CD}^{\circ} 4^{\circ} \mathrm{CD} 16^{\circ *}$ (Q1) & $0.04(0.02-0.06)$ & $0.03(0.02-0.04)$ & $0.02(0.02-0.04)$ & 0.286 & 0.057 \\
\hline $\mathrm{CD}^{\circ}{ }^{\circ} \mathrm{CD}^{\circ}{ }^{\circ}$ (Q2) & $0.02(0.02-0.04)$ & $0.04(0.03-0.06)$ & $0.04(0.03-0.06)$ & 0.001 & $<0.0005$ \\
\hline $\mathrm{CD}^{\circ}{ }^{\circ} \mathrm{CD} 16$ (Q3) & $0.53(0.45-0.66)$ & $0.76(0.63-0.90)$ & $0.79(0.62-1.03)$ & 0.001 & 0.008 \\
\hline
\end{tabular}

Figure 1 Postoperative changes in blood monocyte subsets. (A) Serial flow cytometry plots from a single representative patient, illustrating the quantification strategy (as outlined in the Methods section). Mononuclear cells were obtained from whole blood subjected to dextran sedimentation and discontinuous percoll gradients. The monocyte population was identified by gating on characteristic forward scatter and side scatter appearances. Within this gate, HLA-DR expressing cells were identified, and in this population staining for CD14 and CD16 defined 'classical' CD14++CD16- monocytes (Q3, bottom right quadrant), 'intermediate' CD14++CD16+ monocytes (Q2, top right quadrant) and 'non-classical' CD14+CD16++ monocytes (Q1, top left quadrant). As an additional quality control, expression of CCR2 and $\mathrm{CX}_{3} \mathrm{CR} 1$ was assessed, to confirm that the expected expression pattern of these receptors within each monocyte subset was observed. (B) Serial monocyte subset counts in blood. Median values are shown with the IQR in brackets. Paired t tests were used to determine postoperative change, $n=39$ preoperatively, $n=38$ at $24 \mathrm{~h}$ and $n=27$ at $48 \mathrm{~h}$.

monocyte subsets, ${ }^{30}$ and has been implicated in driving monocyte deactivation, ${ }^{31}$ which in turn is associated with increased risk of infection. ${ }^{12-15}$

Our data also suggested that higher preoperative total cell counts in BAL (predominantly macrophages) and higher levels of circulating neutrophils are associated with a higher risk of postoperative pneumonia. None of our patients had infection detected in BAL samples preoperatively. It is tempting to speculate that patients with a relatively heightened systemic and pulmonary innate immunity in the preoperative phase are more susceptible to the 'second hit' of surgery, and/or to low-level postoperative infective inocula, especially if their monocytes tend to yield a relatively anti-inflammatory profile to inflammatory stimuli. Interestingly, our data did not suggest that neutrophil phagocytosis contributed to risk of infection. While the change in neutrophil phagocytosis postoperatively was statistically significant, and consistent with observations after abdominal surgery, ${ }^{32}$ the levels of phagocytosis reported here are well within the normal range. This is in striking contrast to the situation in critically ill 
Table 3 Postoperative changes in BAL fluid

\begin{tabular}{|c|c|c|c|}
\hline & Preoperative BALF & Postoperative BALF & p Value \\
\hline Total Protein, $\mu \mathrm{g} / \mathrm{mL}$ (IQR) & $118(68.0-188)$ & 187 (94.3-264) & 0.029 \\
\hline Leucocyte count, millions/mL (IQR) & $0.10(0.07-0.17)$ & $0.18(0.09-0.31)$ & 0.012 \\
\hline Macrophages/monocytes, \% (IQR) & $93(75-96)$ & $84(45-92)$ & 0.232 \\
\hline Neutrophils, \% (IQR) & $4(2-23.5)$ & $13(3-49)$ & 0.176 \\
\hline Lymphocytes, \% (IQR) & $2(1-3)$ & $3(1-4)$ & 0.259 \\
\hline Other leucocyte, \% (IQR) & $<1(0)$ & $<1(0)$ & 1 \\
\hline IL-10, pg/mL (IQR) & $0(0.1-0.99)$ & $0.77(0-2.07)$ & 0.018 \\
\hline 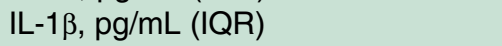 & $1.14(0-7.59)$ & $6.22(1.50-24.58)$ & 0.002 \\
\hline IL-6, pg/mL (IQR) & $4.67(1.08-10.71)$ & $17.71(4.29-45.7)$ & $<0.0005$ \\
\hline IL-8, pg/mL (IQR) & $114(61.6-275)$ & $258(95.7-733)$ & 0.017 \\
\hline
\end{tabular}

cohorts of patients, where reduced neutrophil phagocytosis $(<50 \%$ of neutrophils ingesting 2 or more zymosan particles) is independently associated with increased risk of nosocomial infection. ${ }^{33}$

A unique opportunity to study early innate responses in the lung was also afforded by this study. Previous studies have described pulmonary inflammation after thoracic surgery using serial BAL. ${ }^{34-36}$ However, they have generally been confounded by short intervals between sampling, and by relatively small volumes of instillate. The present study overcame these limitations. Our results confirm that the lung contralateral to the operated tumour is inflamed extremely early in the course of thoracic surgery (ie, within the first couple of hours), with increased neutrophil influx and protein leak across the alveolar-capillary membrane. This effect may be partly attributable to one lung ventilation, which has been shown to induce inflammatory effects in the lung. ${ }^{35}$ Onelung ventilation was used in all patients in this study. Interestingly, only the preoperative BAL cell count

Table 4 Associations with postoperative pneumonia

\begin{tabular}{|c|c|c|c|}
\hline \multicolumn{4}{|l|}{ Panel A } \\
\hline Marker & Pneumonia & No pneumonia & p Value \\
\hline Preoperative leucocyte count in blood $\left(\times 10^{9} / \mathrm{L}\right)$ & $9.1(8.4-11.2)$ & $7.9(6.7-9.5)$ & 0.007 \\
\hline Preoperative blood neutrophils $\left(\times 10^{9} / \mathrm{L}\right)$ & $5.8(5.1-6.9)$ & $4.7(4.1-5.7)$ & 0.015 \\
\hline Preoperative BAL leucocyte count (millions $/ \mathrm{mL}$ ) & $0.14(0.1-0.2)$ & $0.08(0.0-0.1)$ & 0.012 \\
\hline Serum IL-8 (48 h; pg/mL) & $17.4(13.2-21.1)$ & $14.5(10.5-16.8)$ & 0.031 \\
\hline Serum TNF (48 h; pg/mL) & $6.9(4.0-10.3)$ & $7.5(5.3-9.2)$ & 0.023 \\
\hline Monocyte supernatant IL-12 (preoperative; $\mathrm{pg} / \mathrm{mL} / \mu \mathrm{g}$ ) & $0.140(0.1-0.2)$ & $0.187(0.1-0.3)$ & 0.008 \\
\hline 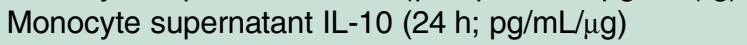 & $3.2(2.1-4.7)$ & $1.4(0.5-2.4)$ & 0.014 \\
\hline 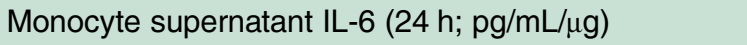 & $525(347-823)$ & $298(115-532)$ & 0.040 \\
\hline 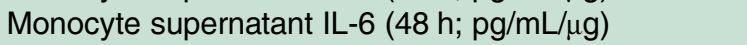 & $616(397-778)$ & $315(176-607)$ & 0.014 \\
\hline Monocyte supernatant IL-8 (48 h; pg/mL/ug) & 2525 (2127-3448) & $2298(1043-2719)$ & 0.040 \\
\hline
\end{tabular}

\section{Panel B}

\begin{tabular}{llllll}
\hline Biomarker & Optimum & Fisher & & & \\
Cut point & p Value & RR (95\% Cl) & Positive LR & Negative LR \\
\hline Pre-op blood neutrophils $\left(\times 10^{9} / \mathrm{L}\right)$ & 5.04 & 0.02 & $3.3(1.1$ to 10.1$)$ & $2.04(1.17-3.57)$ & $0.32(0.11-0.99)$ \\
Pre-op BAL white cell count $(\mathrm{millions} / \mathrm{mL})$ & 0.104 & 0.0093 & $3.4(1.3$ to 9.0$)$ & $2.55(1.25-5.19)$ & $0.40(0.17-0.94)$ \\
Pre-op monocyte supernatant IL-12 $(\mathrm{pg} / \mathrm{mL} / \mu \mathrm{g})$ & 0.147 & 0.0168 & $3.0(1.2$ to 7.3$)$ & $2.78(1.25-6.20)$ & $0.46(0.22-0.96)$ \\
Monocyte supernatant IL-10 $(24 \mathrm{~h} ; \mathrm{pg} / \mathrm{mL} / \mathrm{\mu g})$ & 1.988 & 0.0072 & $4.1(1.3$ to 12.3$)$ & $2.55(1.35-4.83)$ & $0.31(0.11-0.87)$ \\
Monocyte supernatant IL-6 $(24 \mathrm{~h} ; \mathrm{pg} / \mathrm{mL} / \mu \mathrm{g})$ & 414 & 0.02 & $3.1(1.2$ to 8.1$)$ & $2.32(1.19-4.51)$ & $0.41(0.17-0.98)$ \\
\hline
\end{tabular}

(A) Factors associated with postoperative pneumonia. Data are presented as medians with IQR in brackets. Statistical analysis was performed using an unpaired Student $t$ test. Only factors that were significantly different in the two groups are shown. $n=40$ for all comparisons except preoperative BAL, serum and monocyte supernatant experiments at $48 \mathrm{~h}$, where $\mathrm{n}=38$.

(B) Predictive strength of biomarkers associated with pneumonia. The optimum cut point to dichotomise each parameter was determined by Youden's index. $n=40$ for all comparisons except preoperative BAL where $n=38$.

For both (A) and (B), monocyte supernatant values refer to monocytes stimulated ex vivo with LPS.

BAL, Bronchoalveolar lavage; LPS, lipopolysaccharide; LR, likelihood ratio; Pre-op, preoperative; RR, relative risk; TNF, tumour necrosis factor. 
appeared to predict postoperative pneumonia (table 4 panel B), and it remains to be determined whether the postoperative inflammatory response in the lung is exaggerated in patients who have a high preoperative cell count in BAL fluid.

We should emphasise again that our analysis of factors associated with postoperative pneumonia in this population was exploratory and hypothesis generating. As described above, the apparent association with the IL-10 release from monocytes is intriguing, but it must be emphasised that this is an isolated finding. There was not a frank CARS response in these patients. The inevitable conclusions are, first, that the contribution of, and dynamic interplay between, proinflammatory and antiinflammatory cascades are likely to contribute to the pulmonary inflammation observed, and second, that our findings require validation in larger and multicentre cohorts. However, we believe they represent an important first step in this regard, and add to the list of factors potentially associated with complications after thoracic surgery, such as low diffusing capacity for carbon monoxide, certain gene polymorphisms and inflammatory parameters. ${ }^{37-39}$ Ultimately, this work was based on the expectation that a greater understanding of the innate immune processes associated with postoperative complications may allow stratified approaches to management (based on the expectation of postoperative risk) and/or the evolution of novel strategies for the prevention of complications through manipulation of the innate immune response.

When considering our data on postoperative risk, it must be kept in mind that our definition of 'pneumonia' is in the strict sense of 'alveolar inflammation', and the radiological consolidation could represent infiltration of inflammatory cells without infection. We did not judge it ethical to request a further routine BAL for microbiological culture in recovering surgical patients. Nevertheless, our criteria for pneumonia were predefined, alveolar shadowing on CXR was judged by an experienced thoracic radiologist, and 8 of the 14 patients with 'pneumonia' did have bacterial pathogens isolated from the sputum as part of their routine clinical care. Therefore, while we cannot be certain that all patients with 'pneumonia' had bacterial infection, we can be confident that they had alveolar inflammation. It is also important to emphasise that all patients with pneumonia made a good recovery.

In addition, a number of potential limitations should be considered when interpreting our data. Our sample size was small, as we deliberately concentrated on what we believe to be by far the most comprehensive and detailed serial analysis within individual patients in this setting. Our findings provide initial insights but require confirmation in larger cohorts. Also, most of our patients had lung cancer, a burden of tobacco exposure and variable medications including statins, all of which may potentially influence innate immunity. Nevertheless, these factors are likely to be characteristic of, and relatively consistent in, populations of patients being worked up for lung cancer resection, and so the data remain relevant in that specific context. Furthermore, as with all functional studies using neutrophils and monocytes, we cannot be certain that the ex vivo monocyte responsiveness data are representative of biological activity in vivo. In addition, our protocol did not mandate a standardised anaesthetic approach in all patients (anaesthesia was entirely at the discretion of the anaesthetic team). While we are unaware of any significant variations in anaesthetic practice, potential variations could theoretically impact particularly on our BAL data.

In summary, thoracic surgery mobilises circulating classical and intermediate monocytes, and is associated with the release of proinflammatory cytokines from blood monocytes in response to inflammatory stimuli. Monocyte responsiveness to LPS and preoperative levels of leucocytosis appears to be associated with a risk of postoperative pneumonia, but this requires further characterisation and confirmation in larger cohorts.

\section{Author affiliations}

${ }^{1}$ The University of Edinburgh/Medical Research Council Centre for Inflammation Research, The Queen's Medical Research Institute, Edinburgh, UK

${ }^{2}$ Department of Thoracic Surgery, The Royal Infirmary of Edinburgh, Edinburgh, UK

${ }^{3}$ Centre for Population Health Sciences, The University of Edinburgh, Medical School, Edinburgh, UK

${ }^{4}$ Department of Anaesthesia, University of Cambridge, Cambridge Biomedical Campus, Hills Road, Cambridge, UK

${ }^{5}$ Department of Radiology, The Royal Infirmary of Edinburgh, Edinburgh, UK

${ }^{6}$ Institute of Cellular Medicine, Medical School, Newcastle University, Newcastle upon Tyne, UK

Contributors In addition ROJ designed the study, obtained clinical samples, performed experiments, analysed the data and wrote the manuscript. MB and ACM performed experiments and contributed to the writing of the manuscript. NHA designed and performed statistical analysis and contributed to the writing of the manuscript. JTM independently reviewed chest radiographs and contributed to the writing of the manuscript. WSW and AJS designed the study, analysed the data and wrote the manuscript.

Funding The project was funded by a Scottish Chief Scientist's Office research grant (CZB/4/604) and by the Sir Jules Thorn Charitable Trust. In addition, ROJ was supported by the Thoracic Surgery Unit Charitable Funds, Royal Infirmary of Edinburgh.

Competing interests AJS has received funds to attend conferences, in relation to travel, registration and accommodation, from GlaxoSmithKline and Astra Zeneca. He has given non-promotional talks for GSK. He is the chief investigator on a grant on which Becton Dickinson Biosciences are co-applicants and is also a principal investigator on grants where the chief investigator is employed by Becton Dickinson Biosciences.

Ethics approval The study was approved by the Local Research Ethics Committee (LREC No: 09/S1101/11).

Provenance and peer review Not commissioned; externally peer reviewed.

Data sharing statement No additional data are available.

Open Access This is an Open Access article distributed in accordance with the Creative Commons Attribution Non Commercial (CC BY-NC 4.0) license, which permits others to distribute, remix, adapt, build upon this work noncommercially, and license their derivative works on different terms, provided 
the original work is properly cited and the use is non-commercial. See: http:// creativecommons.org/licenses/by-nc/4.0/

\section{REFERENCES}

1. Balk RA. Systemic inflammatory response syndrome (SIRS): where did it come from and is it still relevant today? Virulence 2014;5:20-6.

2. Bone RC. Sir Isaac Newton, sepsis, SIRS, and CARS. Crit Care Med 1996;24:1125-8.

3. Hotchkiss RS, Monneret G, Payen D. Immunosuppression in sepsis: a novel understanding of the disorder and a new therapeutic approach. Lancet Infect Dis 2013;13:260-8.

4. Rosseau S, Hammerl P, Maus U, et al. Phenotypic characterization of alveolar monocyte recruitment in acute respiratory distress syndrome. Am J Physiol Lung Cell Mol Physiol 2000;279:L25-35.

5. Wasserman K, Subklewe M, Pothoff G, et al. Expression of surface markers on alveolar macrophages from symptomatic patients with HIV infection as detected by flow cytometry. Chest 1994;105:1324-34.

6. Krombach F, Gerlach JT, Padovan C, et al. Characterization and quantification of alveolar monocyte-like cells in human chronic inflammatory lung disease. Eur Respir J 1996;9:984-91.

7. Frankenberger M, Eder C, Hofer $\mathrm{P}$, et al. Chemokine expression by small sputum macrophages in COPD. Mol Med 2011:17:762-70.

8. Sunderkötter C, Nikolic T, Dillon MJ, et al. Subpopulations of mouse blood monocytes differ in maturation stage and inflammatory response. J Immunol 2004;172:4410-17.

9. Ziegler-Heitbrock L, Ancuta $\mathrm{P}$, Crowe $\mathrm{S}$, et al. Nomenclature of monocytes and dendritic cells in blood. Blood 2010;116:e74-80.

10. Auffray C, Sieweke MH, Geissmann F. Blood monocytes: development, heterogeneity, and relationship with dendritic cells. Annu Rev Immunol 2009;27:669-92.

11. Geissmann F, Manz MG, Jung S, et al. Development of monocytes, macrophages, and dendritic cells. Science 2010;327:656-61.

12. Döcke WD, Randow F, Syrbe U, et al. Monocyte deactivation in septic patients: restoration by IFN-gamma treatment. Nat Med 1997;3:678-81.

13. Höflich C, Volk HD. Immunomodulation in sepsis. Chirurg 2002;73:1100-4.

14. Caille V, Chiche JD, Nciri N, et al. Histocompatibility leukocyte antigen-D related expression is specifically altered and predicts mortality in septic shock but not in other causes of shock. Shock 2004:22:521-6.

15. Landelle $\mathrm{C}$, Lepape $\mathrm{A}$, Voirin $\mathrm{N}$, et al. Low monocyte human leukocyte antigen-DR is independently associated with nosocomial infections after septic shock. Intensive Care Med 2010;36:1859-66.

16. Stéphan F, Boucheseiche S, Hollande J, et al. Pulmonary complications following lung resection: a comprehensive analysis of incidence and possible risk factors. Chest 2000;118:1263-70.

17. Uramoto $\mathrm{H}$, Nakanishi $\mathrm{R}$, Fujino $\mathrm{Y}$, et al. Prediction of pulmonary complications after a lobectomy in patients with non-small cell lung cancer. Thorax 2001;56:59-61.

18. Baudouin SV. Lung injury after thoracotomy. Br J Anaesth 2003;91:132-42.

19. Haslett C, Guthrie LA, Kopaniak MM, et al. Modulation of multiple neutrophil functions by preparative methods or trace concentrations of bacterial lipopolysaccharide. Am J Pathol 1985;119:101-10.
20. Conway Morris A, Kefala K, Wilkinson TS, et al. C5a mediates peripheral blood neutrophil dysfunction in critically ill patients. Am J Respir Crit Care Med 2009;180:19-28.

21. Falcoz PE, Conti M, Brouchet L, et al. The Thoracic Surgery Scoring System (Thoracoscore): risk model for in-hospital death in 15,183 patients requiring thoracic surgery. $J$ Thorac Cardiovasc Surg 2007;133:325-32.

22. Bernard GR, Artigas A, Brigham KL, et al. Report of the American-European consensus conference on ARDS: definitions, mechanisms, relevant outcomes and clinical trial coordination. The Consensus Committee. Intensive Care Med 1994;20:225-32.

23. Craig SR, Leaver HA, Yap PL, et al. Acute phase responses following minimal access and conventional thoracic surgery. Eur $J$ Cardiothorac Surg 2001;20:455-63.

24. Takenaka K, Ogawa E, Wada H, et al. Systemic inflammatory response syndrome and surgical stress in thoracic surgery. J Crit Care 2006;21:48-55

25. Yamada T, Hisanaga M, Nakajima $Y$, et al. Serum interleukin-6, interleukin-8, hepatocyte growth factor, and nitric oxide changes during thoracic surgery. World J Surg 1998;22:783-90.

26. Urra X, Villamor N, Amaro S, et al. Monocyte subtypes predict clinical course and prognosis in human stroke. J Cereb Blood Flow Metab 2009;29:994-1002.

27. Gordon S, Taylor PR. Monocyte and macrophage heterogeneity. Nat Rev Immunol 2005;5:953-64.

28. Robbins CS, Swirski FK. The multiple roles of monocyte subsets in steady state and inflammation. Cell Mol Life Sci 2010;67: 2685-93.

29. Cabié A, Fitting C, Farkas JC, et al. Influence of surgery on in-vitro cytokine production by human monocytes. Cytokine 1992;4: 576-80.

30. Skrzeczyńska-Moncznik J, Bzowska M, Loseke S, et al. Periphera blood CD14highCD16+ monocytes are main producers of IL-10. Scand J Immunol 2008;67:152-9.

31. Sfeir T, Saha DC, Astiz M, et al. Role of interleukin-10 in monocyte hyporesponsiveness associated with septic shock. Crit Care Med 2001;29:129-33.

32. Kawasaki T, Ogata M, Kawasaki C, et al. Effect of epidural anaesthesia on surgical stress-induced immunosuppression during upper abdominal surgery. Br J Anaesth 2007;98:196-203.

33. Conway Morris A, Brittan M, Wilkinson TS, et al. C5a-mediated neutrophil dysfunction is RhoA-dependent and predicts infection in critically ill patients. Blood 2011;117:5178-88.

34. Schilling $T$, Kozian A, Huth $C$, et al. The pulmonary immune effects of mechanical ventilation in patients undergoing thoracic surgery. Anesth Analg 2005;101:957-65.

35. Breunig A, Gambazzi F, Beck-Schimmer B, et al. Cytokine and chemokine responses in the lungs, pleural fluid and serum in thoracic surgery using one-lung ventilation. J Inflamm (Lond) 2011:8:32.

36. Moloney ED, Mumby SE, Gajdocsi R, et al. Exhaled breath condensate detects markers of pulmonary inflammation after cardiothoracic surgery. Am J Respir Crit Care Med 2004;169:64-9.

37. Ferguson MK, Little L, Rizzo L, et al. Diffusing capacity predicts morbidity and mortality after pulmonary resection. $J$ Thorac Cardiovasc Surg 1988;96:894-900.

38. Shaw AD, Vaporciyan AA, Wu X, et al. Inflammatory gene polymorphisms influence risk of postoperative morbidity after lung resection. Ann Thorac Surg 2005;79:1704-10.

39. Amar D, Zhang H, Park B, et al. Inflammation and outcome after general thoracic surgery. Eur J Cardiothorac Surg 2007;32:431-4. 